\section{Khat use and users readiness to quit khat, qualitative research in the case of street people in Addis Ababa}

\author{
Alembante Fikadu Lemma1*, Urban Robert ${ }^{2}$ and Lajtai Laszlo ${ }^{3}$ \\ ${ }^{1}$ Doctoral Student, ELTE, Faculty of Education and Psychology, Institute of Psychology, Program of \\ Clinical Psychology and Addictions, Hungary \\ 2Professor, Head of Department of Personality and Health Psychology, ELTE, PPK, Hungary \\ ${ }^{3}$ Assistant Professor, Department of Clinical Psychology and Addiction, ELTE, PPK, Hungary
}

\section{Abstract}

Khat is a huge green plant that thrives at high elevations throughout the region ranging from southern to eastern Africa, and in the Arabian Peninsula. However, chewing Khat became common among the young (youth). The objectives of this study were to investigate the khat use behavior, users' self-understanding, and their readiness of stopping using khat among street people in Addis Ababa, Ethiopia. A Semi-structured interview was used to collect information from street people in Addis Ababa. 15 participants were individually interviewed and 11 of them were males and the rest 4 were female participants. The data acquired from the interview was analyzed using descriptive and thematic analysis. Chewing Khat was identified as a common activity among the youth living in the streets of Addis Ababa. Most of the participants have an awareness of the use of khat and its effects on their health but they are still struggling to stop it. While the readiness to stop using khat was investigated and the addiction behavior and the lifestyle of the participants were affecting them from stooping chewing Khat.
More Information

*Address for Correspondence: Alembante Fikadu Lemma, Doctoral Student, ELTE, Faculty of Education and Psychology, Institute of Psychology, Program of Clinical Psychology and Addictions, Hungary, Email: lemma.alembante.fikadu@ppk.elte.hu

Submitted: December 06, 2021

Approved: January 05, 2022

Published: January 06, 2022

How to cite this article: Lemma AF, Robert $U$ Laszlo L. Khat use and users readiness to quit khat, qualitative research in the case of street people in Addis Ababa. J Addict Ther Res. 2022; 6: 001-006.

\section{DOI: 10.29328/journal.jatr.1001020}

ORCiD: orcid.org/0000-0002-0955-3408

Copyright License: (c) 2022 Lemma AF, et al. This is an open access article distributed under the Creative Commons Attribution License, which permits unrestricted use, distribution, and reproduction in any medium, provided the original work is properly cited.

Keywords: Khat; Street people; Addiction; Qualitative research

Check for updates

OPEN ACCESS

\section{Background}

In Sub-Saharan Africa, most of the countries are undergoing significant financial, social, and cultural transformations, which has produced a favorable environment for enlarged and socially destructive drug and alcohol use [1]. Misuse of psychoactive substances is on the rise in Ethiopia, as it is in many other developing countries. Alcohol and khat are the most often abused substances [2].

Substance abuse among Ethiopian adolescents is on the rise, according to studies [3,4]. Students in university and college are the most vulnerable segment of the Ethiopian population when it comes to substance abuse. New prospects, independence from parental control, making a decision, and peer pressure to use or misuse alcohol or extra drugs are all common benefits of attending university [5].

Khat (Catha edulis) is a tall green plant that grows at high elevations in Ethiopia, Somalia, Kenya, Malawi, Uganda, Tanzania, Congo, Zambia, Zimbabwe, Afghanistan, Yemen, and Madagascar, primarily in Ethiopia, Somalia, Kenya, Malawi,
Uganda, Tanzania, Congo, Zambia, Zimbabwe, Afghanistan, Yemen, and Madagascar [6].

Catha edulis, or khat, is a psychostimulant herb used by about 10 (ten) million people every day, primarily in the Middle East and eastern Africa [7]. In the past, khat was considered a Kenyan ceremonial plant, chewed largely by elderly males on social occasions [8].

On average, 100 grams of young khat shrubberies contain 36 milligrams of cathinone, 120 milligrams of cathine, and 8 milligrams of norephedrine [9]. The psychoactive action of khat is assumed to be due to 2 (two) phenylalkylamines called cathine and cathinone, which are chemically similar to amphetamine [10].

Khat is made up of psychoactive substances that impact people's consciousness, behavior, emotion, and thought processes [11]. These active substances have the capability of causing physical and psychological dependence $[11,12]$. The alkaloid amphetamines (cathinone and cathine) found in khat leaves are mostly natural [11]. 
Internationally, the Kat chewers are estimated from 5 to 10 million people, the majority of whom live in the Arabian Peninsula and the Horn of Africa, precisely in the khat belt countries of Ethiopia, Somalia, and Yemen. According to reports, $80 \%-90 \%$ of adult males and $10 \%-60 \%$ of adult females in East Africa ingest khat daily $[9,13]$.

In many parts of the eastern corridor, chewing khat has social, religious, and, cultural benefits. Khat is utilized for a variety of gatherings, community conversations, and conversations. Khat is always employed to accelerate the process during such occasions [14].

Khat chewing has a long history in Ethiopia, dating back to the fourteenth century [6]. While a typical khat chewing session may last between 3-7 hours, cathinone is released within 15-45 minutes of the onset of the chewing $[9,15]$.

While chewing khat, users report feeling as though they can think more clearly and quickly.

Furthermore, khat users registered greater attentiveness, improved ability to concentrate, friendliness, happiness, and a free exchange of ideas as acute effects of the khat. Within 2 hours, this is normally accompanied by a sense of dread, numbing, loss of focus, sluggishness, and insomnia, as well as extreme stress, anxiety, mental instability, irritability, and restlessness [16].

Henceforth, this qualitative study is then, an attempt to investigate the khat use behavior, users' understanding and, perception towards Khat use, and their readiness of stopping using khat about young people who live in the streets of Addis Ababa, the capital of Ethiopia.

\section{Methods}

From then, a descriptive qualitative investigation was carried out between February to March 2021 in Addis Ababa street residents to explore the Khat using behavior and their readiness to quit Khat use.

The study was designed to have some understanding among serious Khat users (Chewers) and to have their perceptions on their readiness to stop using Khat.

This qualitative study included a purposive sampling to select participants who are serious khat users whose lives are dependent on the Streets of Addis Ababa. The participants were given an orientation about the objectives of the study which is to gather information about the issue of Khat Use behaviors in the street living homeless people.

The data collection method was applied in this qualitative study in-depth interview.

The participants of the study were men and women Khat Users with the age range of 21 to 35 years. The inclusion criteria were adults who are serious khat users (chewers) and who are living in the streets of Addis Ababa.

\section{Ethical consideration}

All the participants were informed verbally (verbal Informed Consent). During this process, the participants of the study were informed about their rights and they can stop their participation when they need to terminate their participation in the study at any time, and this can assure their right to participate in the in-depth interview process of the study. This research was approved by Mekelle University, College of Health Sciences, Health Research Ethics Review Committee (HRERC).

The interviewer and facilitator of the study signed a code of conduct to ensure participants of the study and the data integrity process of the study. All the participants were given anonymous identification.

\section{Data collection}

Semi-structure Interview questions were developed for Khat use behaviors and users' perception towards their readiness to quit or stop Chewing Khat.

The items included in the semi-structured interview are

1. How did you start Khat?

2. Has Khat chewing become continues in your life?

3. Do other relevant people understand your Khat chewing behavior?

4. What are the effects of Khat chewing that you face? Health, psychological, social, and personal effects.

5. Are you ready to quit your khat use behavior?

Two interviewers were used to collect information from participants. These two interviewers are male and female interviewers were selected based on their experiences and know-how about addiction. They have good communication and understanding skills about Khat use behaviors. Both interviewers conducted the interview together in all of the interview sessions with the participants one of them as an interviewer and the other one as the facilitator. And they recorded the interview in an audio recorder and the length of the interviews was from 30 minutes to 50 minutes intervals.

After the interviews were conducted for those who were more severe Khat users used a referral mechanism for further psychosocial supports to visit some rehabilitation centers that are found in Addis Ababa, Ethiopia.

\section{Data analysis}

The audio recordings of the in-depth interview were used in Amharic, the national language of Ethiopia, and then hereafter the researcher (AF) translated the interviews into English and a thematic analysis was applied to analyze the interviews. 


\section{Results}

A total of fifteen (15) participants were interviewed for this qualitative study.

\section{Characteristics of participants}

In total 15 participants from these 11 of them were Male and the rest 4 were Female participants. All of them took individual Interviews. The participants of this study who had the interview ranged from 21 to 35 years of age. The duration of Khat use of the participants ranges from 1 year and six months to 7 years. Specifically, the average of their khat use duration for males was 4.3 years and for Females 3.3 years. Of the participants 10 of them were single, 2 of them separated and the rest 3 of them were married. Those who said they were married are currently living with a partner in the streets, but they don't have a legal marriage certificate. Concerning the religious affiliations of the participants, 9 of them were Orthodox Christians, 4 of them followers protestant, and the rest were Muslims and they are 2 in number. When we see the educational background of the participants 12 of them completed elementary education level (grade 8th) and the rest 3 were high school graduates. All most of the participants were living and working daily (Table 1).

Khat Chewing had become a common activity everywhere, among educated or uneducated, poor or rich, students or professionals, married or singles everywhere people in Ethiopia are chewing Khat. This behavior is believed that help chewers stay awake and active in the actions they do but it has a lot of side effects too.

To quote some of the information which was given by the informants.

\begin{tabular}{|c|c|c|c|}
\hline Biographic related Items & Alternatives & Frequency & Percentage \\
\hline \multirow{2}{*}{ Gender } & Male & 11 & $73.33 \%$ \\
\hline & Female & 4 & $26.67 \%$ \\
\hline \multirow{3}{*}{ Age } & $20-25$ & 4 & $26.67 \%$ \\
\hline & $26-30$ & 7 & $46.66 \%$ \\
\hline & $31-35$ & 4 & $26.67 \%$ \\
\hline \multirow{5}{*}{ Duration of chewing Khat } & Less than 1 year & 2 & $13.33 \%$ \\
\hline & $1-2$ years & 2 & $13.33 \%$ \\
\hline & $2-4$ years & 2 & $13.33 \%$ \\
\hline & $4-6$ years & 6 & $40 \%$ \\
\hline & Greater than 6 years & 3 & $20 \%$ \\
\hline \multirow{2}{*}{ Educational background } & Elementary school & 12 & $80 \%$ \\
\hline & Secondary school & 3 & $20 \%$ \\
\hline \multirow{4}{*}{ Region } & Benishangul & 1 & $6.6 \%$ \\
\hline & Oromia & 6 & $40 \%$ \\
\hline & Amhara & 3 & $20 \%$ \\
\hline & Southern Ethiopia & 5 & $33.33 \%$ \\
\hline \multirow{4}{*}{ Religion } & Orthodox & 9 & $60 \%$ \\
\hline & Muslim & 2 & $13.33 \%$ \\
\hline & Protestant & 4 & $26.66 \%$ \\
\hline & Others & & \\
\hline \multirow{3}{*}{ Marital status } & Single & 10 & $66.66 \%$ \\
\hline & Married & 3 & $20 \%$ \\
\hline & Separated & 2 & $13.33 \%$ \\
\hline
\end{tabular}

The general Harms of Khat chewers are based on the participants' responses to the in-depth interview. To list some

- Participants of the study have experienced healthrelated problems which are directly associated with the Khat Use

- Economic problems: unnecessary cost, inability to save, cost of living, hardships faced

- Social problems, conflict with family members, conflict with spouses because of inabilities to have basic needs expenses

- An average of 3 hours spent in the ceremony chewing Khat

- Alcoholic behaviors getting high to spend the time after feeling high in Khat

- Feeling fatigued

- The khat chewers have the possibility to face other additional addictive behaviors and activities like alcoholic, smoking, drug use

- Engaged in theft and robbery activities to buy Khat and other commodities used for consumptions related to the addictive behaviors of the Khat chewers

\section{Participants reaction towards their Khat use behavior}

"When I got Khat to chew I felt like I have everything in my life. I start to forget about every single incident or situation that made me feel worried. So, I know my Khat chewing behavior, but it also gives me to forget and that's I strive to chew khat every single day" 23 years Male participant.

"Because of Khat, everyone here is living on the highways and roads. There are many occasions that we did not find any food to eat but even though we are hungry khat chewing helps us to forget about our food need then particular I continued chewing khat. I know it has along health effect but what else can i do it is the way to forget things." 28 years Male participant

"When other people see us here, they weaken us because we live on the roads, they have various thinking of how much we are suffering when we live here without having basic needs. But here I can imagine that those people's understanding about the reasons why we are here is quite different from ours. Everyone here knows that we have a unique personality or character compared to those who are living with their parents in a warm house and that is the big deal that we have lost in our lives and then we have to struggle for life. In this struggle we developed a good attachment with the others who live in the streets, I can say that we have a good attachment here, we care about every one of us here." 31 years Male participant.

"I have a part of issues since the behavior that I have using even though I did not get cash to purchase Khat I utilized to look 
remains within the trashes of Khat leaves and its sticks in the city's dustbin. After I got the leftovers it can make my mind relax and calm. I believe that that I have developed mental health problems because of this people don't come near or close to me" 35 years old male participant.

I have faced a lot of problems in my life and my parents sent me out of my home after they found out that I was Chewing Khat and smoking a cigarette with my friends in my neighborhood. At that time what I did was staying in the streets and have some friends there too. Now, I want to stop and be like yesterday but still, I am struggling in my daily life."

About other additional drug uses or psychoactive substances the participants take during or after Chewing Khat, most of the informants of the study have listed some of the activities they repeatedly do after they chewed Khat. Some of the additional consumptions were taking alcoholic drinks, smoking cigarettes, taking Hashish, and most of them responded that during the night when they feel cold they smoke benzine, pour it into a plastic cup and smoke it and after that, it gives them warmth(it becomes hot when they sleep).

\section{Attempting to quit khat chewing}

More than half of the participants of the interview said that they want to stop chewing Khat but their current lifestyle and the living condition they have cannot motivate them to quit chewing khat. But some of the participants were even not sure whether they are going to stop chewing khat or not because their focus was on the current situations and life struggle, they encountered in their day-to-day life.

\section{One participant Quote this}

"even though I want to quit khat chewing it is difficult to live in the streets without any psychoactive stimulant. How can I spend and pass the whole day and night and khat is the only way of getting out from the worry that I have and get some mental relief from stressful moments? And my friends cannot motivate me and accept my ideas of quitting khat chewing and I don't want to lose their friendships" 29 years male participant.

"a female member of the participants said that if she tries to quit chewing Khat, she advances difficulty in sleeping and resting. So, to sleep she has to take khat but in the other way she knows and feels that khat chewing is causing her to lose her appetite." 24 years Female respondent.

"if I did not get khat I feel like overpoweringly unstable, high pressures and I ended up short-tempered. And I lose my mood until I found some khat and felt relaxed. So, for me, it is impossible to quit khat even for a single day" 21 years old female participant.

We can understand this khat quitting issue has a lot to face for the participants who chew khat continuously.

\section{Discussion}

The present study explored the perception and understanding of Khat use and readiness to stop using it. It examined the problem of using khat and becoming addicted to it. The semi-structured interview was used as a good qualitative method to elaborate on street people's lifestyles in Addis Ababa, the capital of Ethiopia.

This study was aimed at investigating and exploration of khat users' understanding and readiness for quitting Khat use. The people who live in the streets of Addis Ababa have their perception and understanding of their behavior of Khat use in their everyday life activities. The basic understanding level of the participants. In most qualitative studies, a small number of people contribute a big amount of data in order to learn more about their social environment, as well as the underlying meanings of their behaviors and culture[17].

\section{Knowledge and perception of khat use}

Some individuals prefer to consume khat openly in the streets, beneath the shade of trees and verandas, rather than behind closed doors. The place where you chew has an impact on your self-esteem and dignity. Some people regard khat to be a degraded substance [18].

Jobless adolescents, higher education students, street youngsters, and drivers were named as the most regular users of Khat by the majority of the participants. All of the participants of this particular study were from the streets, who were jobless and mostly young people.

Because of the continuous difficulties of food shortages in the area, one of the most widely reported effects of khat was that it reduced appetite during chewing. Some habitual khat chewers said that chewing khat had no effect on their hunger. [19]. This is also shared with the result of this study on chewing khat gave the respondents to forget about focusing on looking for food, but it also opposed the idea of losing appetite after they chewed khat. But the loss of appetite was shared with the finding of Zeleke, et al's. Sleeping disorders, tooth discoloration, and loss of appetite, as well as constipation, gastritis, and hypertension, are all reported health effects of khat consumption [20].

The idea of the direct outcome of chewing khat gives the users a good feeling, relaxed, and forgets about the issues and situations that made them feel anxious and stressed. And this impression was backed up by the findings of a study that found khat to be a stimulant that made people feel comfortable, cheerful, relaxed, and attentive, as well as giving them the idea that nothing was impossible [19].

One of the responders explained that he chews Khat because he has nowhere else to spend his free time, and that, "I have nothing to do, with the boring day, except to gather with my friends to chew khat" [18]. This result was also 
shared with this study's result on the issues of friendships and attachments with the people who are chewing Khat, especially in the Khat chewing ceremony.

\section{Additional materials and psychoactive uses}

The additional consumptions of other psychoactive materials were taking alcoholic drinks, smoking cigarettes, taking Hashish. These other extra psychoactive materials were shared with a study conducted in 2013. Those who chew Khat regularly tend to be Smokers and alcoholics. This has an effect on several organs in the body, such as those in the respiratory system [20]. And also, another study goes in line with the results of the application of smoking cigarette and alcohol drinks were used while and after chewing khat. Besides, according to the findings of this study, khat use is linked to cigarette smoking, coffee drinking, and the consumption of alcoholic beverages. In the long run, this resulted in drug dependence and addiction, which are serious societal issues among young people. This discovery is consistent with the findings of a study conducted in Mana district, Jimma zone, southwestern Ethiopia [21]. Smoking Benzine especially at nighttime was the only result that cannot be shared or opposed with other studies and the researcher can say that it was the unique result of extra psychoactive stimulants used by the street khat chewers.

\section{Regarding stopping khat}

Even though participants of this study were aware of the effects of continuous usage of khat can cause a lot of health and psychological problems, some of them were ready to stop chewing khat and the others were out of any information about stopping khat usages. This result was shared with a study conducted in 2016 which stated, despite the fact that khat is a calming substance, practically participants in the FGD and in-depth interviews unanimously agreed that we should decolonize ourselves from khat [18]. Furthermore, one FGD participant stated that it is extremely difficult for us to completely stop chewing Khat and said that "In my view, Khat chewer may stop for a while, but never ceases at all" [18]. Withdrawal effect is another element that impacts the intention to stop chewing Khat, according to certain FGD and in-depth interview participants. When Khat chewers stop chewing Khat, they get a headache and fatigability (Ducac), according to the interview, a male respondent who is 26-yearold, "I would be paralyzed if I don't chew Khat." [18].

Respondents in another study expressed a strong desire to stop or reduce their khat consumption.

Squandering time, mental dependence, the burden of family or companions, the catalytic features of khat (Its proclivity for pressuring you to do things you don't want to do), the damage to one's general health, as well as to one's psychosocial and economic well-being were all cited as important motivators for wanting to quit by the participants [22].

\section{Conclusion}

From the interviewed participants this article has investigated and explored the understanding and readiness towards quitting the suing of khat. According to the perceptions of the participants understanding concerning the use of khat and its effect on their health care was well known but they failed to stop using khat. While the readiness to stop using khat was investigated and the addiction behavior and the lifestyle of the participants were affecting them from stooping chewing Khat.

\section{References}

1. John-langba J. Alcohol, Drug Use, and Sexual-risk Behaviors among Adolescents in Four Sub-Saharan African Countries. In Proceedings of the Annual Meeting Program of the Population Association of America. 2003; 5-8.

2. Fekadu. African Journal of Drug and Alcohol Studies. 2007; 6. 39.

3. Kebede Y. Cigarette smoking and khat chewing among college students in North West Ethiopia. Ethiopian J Health Develop. 2002; 16: $9-17$.

4. Kebede D, Alem A, Mitike G, Enquselassie F, Berhane F, et al. Khat and alcohol use and risky sex behaviour among in-school and out-ofschool youth in Ethiopia. BMC Public Health. 2005; 5: 109. PubMed: https://pubmed.ncbi.nlm.nih.gov/16225665/

5. Deressa W, Azazh A. Substance use and its predictors among undergraduate medical students of Addis Ababa University in Ethiopia. BMC Public Health. 2011; 11: 660.

6. Gebissa E. Khat in the Horn of Africa: Historical perspectives and current trends. J Ethnopharmacol. 2010; 132: 607-614. PubMed: https://pubmed.ncbi.nlm.nih.gov/20227478/

7. Berihu BA, Asfeha GG, Welderufael AL, Debeb YG, Zelelow YB, et al. Toxic effect of khat (Catha edulis) on memory: Systematic review and meta-analysis. J Neurosci Rural Pract. 2017; 8: 30-37. PubMed: https://pubmed.ncbi.nlm.nih.gov/28149078/

8. Carrier N. Bundles of choice: Variety and the creation and manipulation of Kenyan Khat's value. Ethnos. 2006; 71: 415-437.

9. Feyissa AM, Kelly JP. A review of the neuro pharmacological properties of khat. Prog Neuropsychopharmacol Biol Psychiatry. 2006; 32: 1147-1166.

PubMed: https://pubmed.ncbi.nlm.nih.gov/18561890/

10. Kalix $P$, Khan I. Khat: An amphetamine-like plant material. Bull World Health Organ. 1984; 62: 681-686.

PubMed: https://www.ncbi.nlm.nih.gov/pmc/articles/PMC2536214/

11. Wabe NT. Chemistry, pharmacology, and toxicology of khat (catha edulis forsk): a review. Addiction \& Health. 3: 137-149.

PubMed: http://www.ncbi.nlm.nih.gov/pubmed/24494

12. Kalix P. Pharmacological properties of the stimulant khat. Pharmacol Ther. 1990; 48: 397-416.

PubMed: https://pubmed.ncbi.nlm.nih.gov/1982180/

13. Wondemagegn AT, Cheme MC, Kibret KT. Perceived Psychological, Economic, and Social Impact of Khat Chewing among Adolescents and Adults in Nekemte Town, East Welega Zone, West Ethiopia. BioMed Res Int. 2017

PubMed: https://pubmed.ncbi.nlm.nih.gov/28265577/

14. Hailu T. The Conceptualization of Khat, Its Production and Its SocioEconomic Implications in Eastern Ethiopia. Int $\mathrm{J}$ Educat Culture Society. 2019; 4: 54.

15. Banjaw MY, Schmidt WJ. Behavioural sensitisation following repeated intermittent oral administration of Catha edulis in rats. Behav Brain 
Res. 2005; 156: 181-189.

PubMed: https://pubmed.ncbi.nlm.nih.gov/15582104/

16. Cox G, Rampes H. Adverse effects of khat: A review. Advances in Psychiatric Treatment. 2003; 9: 456-463.

17. Neale J, Allen D, Coombes L. Qualitative research methods within the addictions. Addiction. 2005; 100: 1584-1593.

PubMed: https://pubmed.ncbi.nlm.nih.gov/16277621/

18. Estifanos M, Azale T. Intention to Stop Khat Chewing and Associated Factors among Khat Chewers in Dessie City, North Eastern Ethiopia. Epidemiology: Open Access. 2016; 6: 1-11.

19. Teferra S, Hanlon C, Alem A, Jacobsson L. Khat chewing in persons with severe mental illness in Ethiopia: A qualitative study exploring perspectives of patients and caregivers. Transcult Psychiatry. 2011;
48: 455-472.

PubMed: https://pubmed.ncbi.nlm.nih.gov/21911510/

20. Zeleke A, Awoke W, Gebeyehu E, Ambaw F. Khat chewing practice and its perceived health effects among communities of Dera Woreda, Amhara region, Ethiopia. Open J Epidemiol. 2013; 03: 160-168.

21. Jemal A, Badassa WC, Kasahun E, Kyung Ryang K, Chang Soo K. Khat use and its impact on academic performance: The case of Jimma University, Ethiopia. Educational Research and Reviews. 2015; 10: 2084-2095.

22. Mihretu A, Teferra S, Fekadu A. What constitutes problematic khat use? An exploratory mixed methods study in Ethiopia. Subst Abuse Treat Prev Policy. 2017; 12: 17.

PubMed: https://pubmed.ncbi.nlm.nih.gov/28327160/ 